Canadian

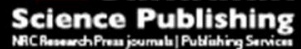

Canadian Journal of Physiology and Pharmacology Revue canadienne de physiologie et pharmacologie

\title{
Effects of baicalin on alveolar fluid clearance and a-ENaC expression in Rats with LPS-induced acute lung injury
}

\begin{tabular}{|r|l|}
\hline Journal: & Canadian Journal of Physiology and Pharmacology \\
\hline Manuscript ID & cjpp-2016-0212.R1 \\
\hline Danuscript Type: & Article \\
\hline Complete List of Authors: & $\begin{array}{l}\text { Deng, Jia; Traditional Chinese medical hospital of Jiangbei District } \\
\text { Wang, Dao-xin; Second Affiliated of Chongqing Medical University } \\
\text { Liang, Ai-ling; Traditional Chinese medical hospital of Jiangbei District } \\
\text { Tang, Jing; Traditional Chinese medical hospital of Jiangbei District } \\
\text { Xiang, Da-kai; Traditional Chinese medical hospital of Jiangbei District }\end{array}$ \\
\hline Keyword: & $\begin{array}{l}\text { baicalin, acute lung injury, alveolar fluid clearance, epithelial sodium } \\
\text { channel }\end{array}$ \\
\hline &
\end{tabular}


Effects of baicalin on alveolar fluid clearance and $\alpha$-ENaC expression in Rats with LPS-induced acute lung injury

${\text { Deng, } \text { Jia }^{1}{ }^{*} \text {; Wang, Dao-xin }}^{2}$; Liang, Ai-ling ${ }^{1}$; Tang, Jing ${ }^{1}$; Xiang, Da-kai ${ }^{1}$

${ }^{1}$ Department of Respiratory Medicine, Traditional Chinese medical hospital of Jiangbei District, Chongqing, China and ${ }^{2}$ Department of Respiratory Medicine, Second Affiliated of Chongqing Medical University, Chongqing, China.

* Corresponding author

Requests for reprints: Deng, Jia; Department of Respiratory Medicine, Traditional Chinese medical hospital of Jiangbei District, Chongqing, China. Tel: +86-23-6773 -5334. E-mail: 2081079756@qq.com

\section{Author contributions:}

Conception and design: Deng, Jia and Wang, Dao-xin Financial support: Deng, Jia

Collection and assembly of Data: Deng, Jia; Liang, Ai-ling

Data analysis and interpretation: Deng, Jia; Liang, Ai-ling; Tang, Jing; Xiang, Da-kai

Manuscript writing: Deng, Jia 


\begin{abstract}
Baicalin has been reported to attenuate lung edema in the process of lung injury. However, the effect of baicalin on alveolar fluid clearance and $\mathrm{ENaC}$ expression has not been tested. SD rats were anesthetized and intratracheally injected with either $1 \mathrm{mg} / \mathrm{kg}$ lipopolysaccharide (LPS) or saline vehicle. Baicalin with various concentrations $(10,50$ and $100 \mathrm{mg} / \mathrm{kg})$ was injected intraperitoneally $30 \mathrm{~min}$ before administration of LPS. Then lungs were isolated for measurement of alveolar fluid clearance (AFC), cyclic adenosine monophosphate (cAMP) level and cellular localization of $\alpha$-epithelial sodium channel $(\alpha$-ENaC). Moreover, mouse alveolar type II (ATII) epithelial cell line was inbubated with baicalin (30uM), adenylate cyclase inhibitor SQ22536 $(10 \mathrm{uM})$ or cAMP-dependent protein kinase inhibitor (PKA) KT5720 (0.3uM) $15 \mathrm{~min}$ before LPS $(1 \mu \mathrm{g} / \mathrm{ml})$ incubation. Protein expression of $\alpha$-ENaC was detected by Western blot. Baicalin increased cAMP concentration and alveolar fluid clearance in a dose-dependent manner in rats with LPS-induced ALI. The raise of alveolar fluid clearance induced by baicalin was associated with a increase in the abundance of $\alpha$-ENaC protein. SQ22536 and KT5720 prevented the increase of $\alpha$-ENaC expression caused by baicalin in vitro. These findings suggest that baicalin prevents LPS-induced reduction of AFC by upregulating $\alpha$-ENaC protein expression, which is activated by stimulating cAMP/PKA signaling pathway.
\end{abstract}

Key word: baicalin, acute lung injury, alveolar fluid clearance, epithelial sodium channel 


\begin{abstract}
Abbreviation: AC, adenylyl cyclase; AFC, alveolar fluid clearance; ALI, acute lung injury; ATII cells, alveolar type II epithelial cells; cAMP, cyclic adenosine monophosphate; CK, cytokeratin; $\mathrm{ENaC}$, epithelial sodium channel; HSC, highly selective cation; LPS, lipopolysaccharide; MAPK, mitogen-activated protein kinase; NSC, non-selective cation; DBcAMP, dibutyryl cAMP; PKA, protein kinase A; RIA, radioimmunoassay.
\end{abstract}




\section{Introduction}

Acute lung injury (ALI) is associated with high morbidity and mortality in critically ill patients. It is a distinct form of acute respiratory failure characterized by diffuse pulmonary infiltrates, progressive hypoxemia, reduced lung compliance and abnormal hydrostatic pressure (Phua et al. 2009; Tang et al. 2009). Until now, there are still no effective therapeutic strategies or pharmacological interventions to treat it. Therefore, novel therapies for ALI are urgently needed. Besides various cytokines induced by lung inflammation (Hassoun et al. 1998; Sato et al. 1998), widespread destruction of the capillary endothelium, extravasations of protein rich fluid and interstitial edema are major complications of ALI (Lucas et al. 2009). Reduced alveolar fluid clearance (AFC) capacity, which is accompanied by pulmonary permeability edema, was observed in a majority of patients in ALI, and maximal AFC was associated with better clinical outcomes (Ware and Matthay 2001). Thus, a therapeutic strategy for recovering the balance between alveolar fluid formation and reabsorption may be an effective treatment for ALI.

Baicalin (5,6-dihydroxyflavone,7-glucuronic acid) is a major compound isolated from the root of Scutellaria baicalensis Georgi used in china to treat infectious diseases. Recent studies indicate that baicalin exerts a multitude of pharmacological activities including inhibition of platelet aggregation (Lee et al. 2015), reduction of endotoxin generation (Liu et al. 2008) and bacteria resistance (Liu et al. 2000). Wang and Liu 2014 and Zhang et al. 2008 have shown that baicalin improved tissue injury in experimental sepsis and multiple organ injury in severe pancreatitis. Moreover, baicalin attenuated air embolism-induced ALI and inhibited inflammatory effects against lipopolysaccharide (LPS)-induced ALI (Ding et al. 2016; Li et al. 2009). As previously demonstrated, baicalin attenuated lung edema in the process of lung injury. However, the detailed mechanism underlying the effect of baicalin on pulmonary edema and alveolar filling is still unknown.

In view of these data, AFC and lung immunocytochemistry were analyzed in the isolated and ventilated lung of LPS-induced ALI firstly. A second set of experiment was performed on alveolar type II epithelial cells (ATII cells) to elucidate the mechanisms of therapeutic effects associated with baicalin exposure to the respiratory system. 


\section{Methods}

\subsection{Materials}

Baicalin, lipopolysaccharide (LPS), amiloride, sodium pentobarbital, evans blue, SQ22536 and KT5720 were all obtained from Sigma (St Louis, MO, USA).

\subsection{Animal model}

All protocols involving rats were approved by the Institutional Review Boards of Chongqing Medical University. Male Sprague-Dawley rats (220-240 g, Beijing Experimental Animal Center) received human care in accordance with the institution's ethical guidelines for the care and use of laboratory animals. SD rats were anesthetized by intraperitoneal administration of sodium pentobarbital $(50 \mathrm{mg} / \mathrm{kg}$ body weight). Experimental rats were intratracheally injected with 1 $\mathrm{mg} / \mathrm{kg}$ LPS (Escherichia coli 055:B5; Sigma, St. Louis, MO) dissolved in $0.3 \mathrm{ml}$ saline, whereas control rats received only saline vehicle $(0.3 \mathrm{ml}$ saline). Baicalin with various concentrations (10, 50 and $100 \mathrm{mg} / \mathrm{kg}$ ) was injected intraperitoneally thirty minutes before administration of LPS. The trachea, lungs and hearts were isolated en bloc. The left lungs were separated to measure lung water volume and cAMP level. The right lungs were prepared to assess alveolar fluid clearance.

\subsection{Lung water content}

After administration of LPS with or without baicalin, blood was drawn and left lung was removed and dried at $95^{\circ} \mathrm{C}$ for 48 hours. Lung water content was estimated by calculating the ratio of the wet lung weight to the dry lung weight (mg) per gram of body weight.

\subsection{Measurement of cAMP in lung}

Lung samples were treated with isobutyryl methylxanthine (Sigma-Aldrich) to inhibit phosphodiesterases, and homogenized in ice-cold $1 \mathrm{M} \mathrm{TCA}$, then centrifuged at $2500 \mathrm{~g}$ to precipitate particulate material. The cyclic adenosine monophosphate (cAMP) content in the supernatant was measured by radioimmunoassay (RIA) as previously described (Seybold et al. 1998).

\subsection{Alveolar fluid clearance}

Alveolar fluid clearance was estimated by measurement of progressive increase in the concentration of alveolar Evans blue dye, as previously described (Sakuma et al. 2004). Briefly, fluid $(1.5 \mathrm{ml})$ containing evans blue-labeled $5 \%$ bovine albumin was instilled into the airway of right lung, and followed by $2 \mathrm{ml}$ oxygen to deliver all the instill solution into the alveolar spaces. Then the lungs were placed into a prewarmed incubator at $37^{\circ} \mathrm{C}$ and inflated at an airway pressure of $7 \mathrm{cmH}_{2} \mathrm{O}$ with $100 \%$ oxygen. After 5 min (time 0 ) and 65 min (time $60 \mathrm{~min}$ ), samples were removed through the catheter by gentle aspiration. The change in concentration of protein in the time $60 \mathrm{~min}$ compared with the time 0 min samples was used to determine the volume of fluid cleared as follows:

$$
\mathrm{AFC}=\left[\left(\mathrm{V}_{\mathrm{i}}-\mathrm{V}_{\mathrm{f}}\right) / \mathrm{V}_{\mathrm{i}}\right] \times 100 \% \quad \mathrm{~V}_{\mathrm{f}}=\left(\mathrm{V}_{\mathrm{i}} \times \mathrm{EB}_{\mathrm{i}}\right) / \mathrm{EB}_{\mathrm{f}}
$$

$\mathrm{V}$ represents the initial volume (i) and final volume (f) of alveolar fluid. EB represents the concentration of Evans blue dye in initial solution (i) and final alveolar fluid(f). 


\subsection{Histological analysis}

Lungs were fixed by immersed into a $10 \%$ formalin solution for a week, and sectioned at $3 \mathrm{~mm}$. These sections were embedded in paraffin then sectioned at $5 \mu \mathrm{m}$ and stained with HE. The morphological changes were examined under light microscopy. All photographs are at $\times 100$ magnification.

\subsection{Immunocytochemistry}

The lung tissue was dehydrated in graded ethanol and left in xylene overnight. Then tissue was embedded in paraffin and cut into $2-\mu \mathrm{m}$ sections on a rotary microtome. Sections had endogenous peroxidase activity blocked with $0.5 \% \mathrm{H}_{2} \mathrm{O}_{2}$ in methanol for $10 \mathrm{~min}$ and boiled in a target retrieval solution ( $1 \mathrm{mmol} / 1$ Tris, $\mathrm{pH} 9.0$, with $0.5 \mathrm{mmol} / 1 \mathrm{EGTA})$ for $10 \mathrm{~min}$. Nonspecific binding was prevented by $50 \mathrm{mmol} / 1 \mathrm{NH}_{4} \mathrm{Cl}$ in PBS for $30 \mathrm{~min}$ followed by PBS blocking buffer (1\% BSA, $0.05 \%$ saponin, and $0.2 \%$ gelatin). The sections were incubated with primary antibody (rabbit anti-epithelial sodium channel antibody, Abcam) at $4{ }^{\circ} \mathrm{C}$. Then the sections were washed and incubated with horseradish peroxidase-conjugated secondary antibody (goat anti-rabbit immunoglobulin, Abcam). After $1 \mathrm{~h}$ of incubation at room temperature, coverslips were mounted with a hydrophilic mounting medium containing anti-fading reagent (N-propyl-gallat, P-3101; Sigma Chemical). Light microscopy was carried out with a Leica DMRE microscope (Leica Microsystems). All photographs are at $\times 400$ magnification. The number of positive cells $s$ was counted in randomly 5 high-power fields of each section and averaged.

\subsection{ATII cell culture}

The mouse alveolar type II (ATII) epithelial cell line (MLE12 cells) was purchased from Yili Bio-technology (Shanghai, China). Lamellar bodies and expression of cytokeratin (CK) 18, CK19, occluding, surfactant protein B and C, which exhibits the characteristics of ATII cells, were observed in this cell line. ATII cells were cultured in high DMEM (Hyclone) with 10\% FBS (GIBCO), 1\% L-glutamine, and a 1\% solution of penicillin and streptomycin. Cells were passaged using $0.25 \%$ trypsin when they reached $80 \%$ confluence, and were diluted $1: 3$ at each passage.

\subsection{Western blot}

Proteins were separated in 10\% SDS-PAGE gels and transblotted onto polyvinyllidene difluoride membrane. After incubation in a blocking solution containing $20 \mathrm{mM}$ Tris-Cl, $\mathrm{pH} 7.5,0.5 \mathrm{M}$ sodium chloride and 5\% nonfat dried milk for $1 \mathrm{~h}$, the membrane was incubated first with antibody at $4^{\circ} \mathrm{C}$ overnight in an antibody buffer containing $20 \mathrm{mM}$ Tris- $\mathrm{Cl}, \mathrm{pH} 7.5,0.5 \mathrm{M}$ sodium chloride, $0.1 \%$ Tween 20 and $0.2 \%$ nonfat dried milk. The membrane was incubated with secondary antibody at room temperature for 1 h. $\alpha$-ENaC (ab96867) and $\beta$-actin (ab8226) polyclonal antibodys were purchased from Abcam (Cambridge, MA, USA). ECL kit (Sigma, USA) was used to develop the image.

\subsection{Statistics}

Summary data are the mean and SD. Student's $t$ test or Fisher ANOVA test was used for statistical comparison between groups. $P<0.05$ was considered as statistically significant. 


\section{Results}

\subsection{Effect of baicalin on lung water content}

The lung water content was examined after 6 hours of administration with LPS or baicalin (Fig. 1). LPS largely increased the lung water contents compared with control group $(P<0.01)$. However, baicalin pre-treatment significantly decreased LPS-induced lung water content to control level $(P<0.05)$.

\subsection{Effect of baicalin on cAMP concentration in lung tissue}

Cyclic adenosine monophosphate (cAMP) was estimated 6 hours after in absence or presence of baicalin by RIA (Fig. 2). Compared with LPS treatment group, cAMP concentration in lung tissue was increased in a dose-dependent manner with increasing doses of baicalin ( $\mathrm{pmol} / \mathrm{g}$, control $669.6 \pm 82.65$, LPS $265.8 \pm 62.39$, baicalin $10 \mathrm{mg} / \mathrm{kg} 381 \pm 60.51$, baicalin $50 \mathrm{mg} / \mathrm{kg} 493.4 \pm 63.93$, baicalin $100 \mathrm{mg} / \mathrm{kg} 582.4 \pm 47.44)$.

\subsection{Effect of baicalin on alveolar fluid clearance}

Rats were treated using increasing doses of baicalin $(10,50$ and $100 \mathrm{mg} / \mathrm{kg})$ by intraperitoneal injection before administration of LPS. AFC was reduced in LPS-treated rats compared with control (Fig. 3). Baicalin pre-treatment significantly increased AFC in a dose-dependent manner.

To further elucidate the mechanisms which baicalin exerts its effects by, amiloride was added to the instillate for AFC measurement (Fig. 4). Alveolar fluid clearance was about $20 \%$ in control group. Addition of amiloride or LPS to the instillate decreased fluid clearance by $87 \%$ and $77 \%$. Compared with ALI group, baicalin $(100 \mathrm{mg} / \mathrm{kg})$ increased fluid clearance by $49 \%$. Baicalin had no significant effect as amiloride was added in the instillate $(p>0.05)$.

\subsection{Effect of baicalin on histological alteration in lung tissue}

Lung tissue specimens were obtained 6 hours after LPS administration, with or without pretreatment with baicalin. Compared to control group (Fig. 5A), interstitial edema and inflammatory cell infiltration were observed in LPS treatment group (Fig. 5B). However, Interstitial edema and inflammatory cell infiltration were markedly decreased when pretreated with baicalin (Fig. 5C).

\subsection{Effect of baicalin on cellular localization of a-epithelial sodium channel ( $\alpha$-ENaC)}

Immunohistochemical analysis revealed that the number of cells expressing $\alpha-\mathrm{ENaC}$ decreased (Fig. 6B) in ALI group induced by LPS vs control (Fig. 6A). In contrast, pretreatment with baicalin increased the number of cells expressing $\alpha$-ENaC (Fig. 6C).

\subsection{Effect of baicalin on a-epithelial sodium channel ( $\alpha-E N a C)$ in vitro}

To clarify the contribution of baicalin to alveolar fluid clearance, we tested the expression of $\alpha$-ENaC in vitro (Fig. 7). LPS $(1 \mu \mathrm{g} / \mathrm{ml})$ incubated group resulted in a markedly decrease in $\alpha$-ENaC protein expression when compared with control $(0.282 \pm 0.07$ vs $0.824 \pm 0.08)$, whereas preincubation of baicalin $(30 \mathrm{uM})$ before was associated with a increase in the abundance of 
$\alpha$-ENaC protein $(0.69 \pm 0.09$ vs LPS). The western analysis in vitro were consistent with immunohistochemical changes in vivo (Fig. 6).

To further validate the mechanism of $\alpha$-ENaC abundance changes, ATII cells were pre-incubated for $15 \mathrm{~min}$ with an adenylate cyclase inhibitor (SQ22536, 10uM) or a cAMP-dependent protein kinase inhibitor (KT5720, 0.3uM) before baicalin was added (Fig. 7). As can be seen, baicalin-induced increases in $\alpha$-ENaC expression was attenuated by SQ22536 (0.41 \pm 0.07 vs LPS+Baicalin), which suggests that baicalin appears to modulate $\alpha$-ENaC expression through cAMP pathway. Moreover, KT5720 prevented the increasing effect of baicalin on $\alpha$-ENaC expression $(0.38 \pm 0.05$ vs LPS + Baicalin). This results indicate that the PKA-dependent signaling pathway is involved in upregulation of $\alpha-\mathrm{ENaC}$ expression caused by baicalin. 


\section{Discussion}

There are three findings of this study. First, baicalin decreased pulmonary edema and increased alveolar fluid clearance in a dose-dependent manner in rats with ALI. Second, the raise of alveolar fluid clearance induced by baicalin was associated with a increase in the abundance of $\alpha$-ENaC protein. Third, baicalin increased cAMP concentration in lung tissue, whereas an adenylate cyclase inhibitor or a cAMP-dependent protein kinase inhibitor prevented the increase of $\alpha$-ENaC expression caused by baicalin. Our data indicate that baicalin produces a increase in alveolar fluid clearance and upregulation of $\alpha$-ENaC protein expression by stimulating the cAMP/PKA pathway in rats with ALI.

Several previous studies demonstrated that baicalin attenuated lung edema in the rats model of air embolism-induced acute lung injury, multiple organ injury in sever acute pancreatitis and sepsis induced by cecal ligation and puncture (Ding et al. 2016; Li et al. 2009; Zhang et al. 2008). Our results showed that baicalin also attenuated lung edema in LPS-induced acute lung injury. In vivo, the volume of alveolar fluid is determined by the balance between its formation and reabsorption, termed alveolar fluid clearance (AFC). In our present study, baicalin pre-treatment increased AFC in a dose-dependent manner in rats with ALI. This was first time to demonstrate the association between baicalin and AFC. Meanwhile, addition of amiloride eliminated the effects of baicalin on AFC. It is implied that the increase effect induced by baicalin is amiloride sensitive. Amiloride is known to be a specific sodium channel blocker and exerts its specific inhibition effect on transepithelial sodium transport by ENaC (Benos et al. 1987). Therefore, we wondered if baicalin played a role in regulation of amiloride-sensitive $\mathrm{ENaC}$ expression. However, there is no study investigating the effect of baicalin on $\mathrm{ENaC}$ expression before. The detailed mechanism underlying the effect of baicalin on pulmonary edema and alveolar filling in rats with ALI needs to be elucidated.

Epithelial sodium channel, which is composed of $\alpha, \beta$, and $\gamma$ homologous subunits, is the main force to drive sodium ions transepithelial reabsorption for driving fluid out of alveolar spaces. Several studies have found that $\mathrm{ENaC}$ is the major determinant of alveolar fluid clearance across the alveolar epithelium (Vivek and Kenneth 2008). In Xenopus laevis oocytes or rat thyroid epithelial cells, only $\alpha$-ENaC presented $\mathrm{Na}^{+}$-dependent current consistent with active $\mathrm{Na}^{+}$transport (Canessa et al. 1993, Snyder 2000). In mice with genetical deficient for $\alpha$-ENaC, newborn mice were unable to clear airway fluid and died within $40 \mathrm{~h}$ of birth (Hummler et al. 1996). These all suggest the critical importance of $\alpha$-ENaC required of $\mathrm{Na}^{+}$transport. In present study, baicalin prevented the inhibitory effect of LPS on the number of cells expressing $\alpha-\mathrm{ENaC}$ in rats with ALI, and reversed the decrease of $\alpha$-ENaC protein expression induced by LPS in vitro. Numberous previous studies have reported that $\alpha$-ENaC may be regulated by LPS, however, these findings are controversial, as both increased and decreased effect in $\alpha$-ENaC expression were observed (Baines et al. 2010; Dodrill and Fedan 2010). Until recently, Daginais et al. 2005 and Sheng et al. 2014 has demonstrated that LPS may modulate $\alpha$-ENaC expression in a biphasic manner, with a transient increase in the early stage and a sustained decrease thereafter. However, the early stage of LPS exertion is not well-defined yet. In this study, $\alpha$-ENaC protein expression was decreased after LPS 
incubation for $6 \mathrm{~h}$. Our results indicated that baicalin increases AFC in rats with ALI via upregulation of $\alpha$-ENaC protein expression. All three subunits $(\alpha, \beta$ and $\gamma)$ constitute the typical $\mathrm{ENaC}$ called highly selective cation (HSC) channel, whereas $\alpha$-ENaC alone constitute the non-selective cation (NSC) channel (Johnson et al. 2006). Although more than 100-fold potentiation in current was observed when three subunits were expressed together, it was a 3-5-fold potentiation in current when cRNAs encoding either $\beta$-ENaC or $\gamma$-ENaC were coinjected with $\alpha$-ENaC cRNA (Lingueglia et al. 1996). Canessa et al. 1994 conclude that $\alpha$-ENaC is sufficient to induce channel activity. Therefore, the upregulation of $\alpha$-ENaC protein expression induced by baicalin in our study actually led to a increase in the quantity of active epithelial sodium channels. It is worthy to consider that following treatment with baicalin there may be additional $\mathrm{ENaC}$ which is at least composed of just the $\alpha$-subunit.

To further elucidate the mechanism responsible for regulation of $\alpha$-ENaC protein expression by baicalin, we estimated the cAMP level in lung. Baicalin increased the cAMP concentration in lung tissue in a dose-dependent manner. In addition, $\alpha$-ENaC protein expression was decreased in response to SQ22536 pre-incubation, which is an adenylate cyclase inhibitor, before baicalin was added in vitro. Sevral previous studies (Dagenais et al. 2001; Minakata et al. 1998) demonstrate that $\alpha$-ENaC mRNA expression in alveolar epithelial cells is upregulated when exposed to dibutyryl cAMP (DBcAMP). However, the protein expression of $\alpha$-ENaC was not evaculated in these studies. The association of changes in $\alpha$-ENaC mRNA between protein is unclear. Our results suggest an cAMP-mediated increase in $\alpha$-ENaC protein expression induced by baicalin. Other study was in line with our results. In human lung H441 cell, baicalin induced differential expression of cytocheome $c$ oxidase, and elevated cAMP consequently (Cheng et al. 2003). cAMP is a key second messenger in amiloride-sensitive ENaC-mediated sodium reabsorption. Previous studies in vivo and vitro indicate that the effects of cAMP on $\alpha$-ENaC include upregulation of $\alpha$ subunit expression, translocation to and increased expression at the apical membrane, and promotion of $\mathrm{Na}^{+}$transport (Morris and Schafer 2002; Planès and Caughey 2007; Thomas et al. 2004).

Receptor activation contributes to the generation of cAMP via the stimulation of adenylyl cyclase (AC) by the G-protein subtype Gs, which results in the activation of protein kinase A (PKA). PKA is responsible for regulatory effects on cellular functions through the phosphorylation of specific target proteins. Evidence from mesenteric artery demonstrated that baicalin produces activation of ion channels such as $\mathrm{Ca}^{2+}$-activated $\mathrm{K}^{+}$channel by stimulating the cAMP/PKA pathway (Lin et al. 2010). Our present findings indicate that KT5720, a cAMP-dependent protein kinase inhibitor, prevented the increase of $\alpha$-ENaC induced by baicalin. Thus, cAMP/PKA-dependent pathway play a role in baicalin-induced upregulation of $\alpha$-ENaC. In addition, we observed that in presence of $\mathrm{KT} 5720, \alpha-\mathrm{ENaC}$ protein level was decreased, but not entirely abolished. An earlier study suggested that there was no consensus cAMP responsive element in the rat $\alpha$-ENaC promotor (Otulakowski et al. 1999). Intermediary cAMP-binding proteins may provide an alternative pathway for cAMP to stimulate other signaling cascade pathways besides activating PKA (Vossler et al. 1997). Studies by Mustafa et al. 2008 and Richards et al. 2001 demontrated that increased intracellular cAMP levels regulates other signaling pathways such as mitogen-activated protein kinase (MAPK) pathway, and cross talk between PKA and MAPK pathways contributes to 
increased level of $\alpha-\mathrm{ENaC}$.

There are several limitations resulting from our study. First, electrogenous activity analysis of $\mathrm{ENaC}$ was not carried out in the study. Second, since the critical importance of $\alpha$-ENaC for $\mathrm{Na}^{+}$ transport, we only tested the changes in abundance of $\alpha$-ENaC without other two subunits ( $\beta$ and $\gamma)$. Third, alveolar fluid clearance regulated by baicalin and other agents was tested in the isolated lungs, the results could not be directly compared with the application of the agents in vivo. 


\section{Conclusions}

In summary, our present findings provide the first evidence that baicalin prevents LPS-induced reduction of AFC by upregulating $\alpha$-ENaC protein expression. Also, we have shown that baicalin elevates intracellular cAMP concentration, and cAMP/PKA pathway contributes toward biacalin-induced upregulation of $\alpha$-ENaC. Based on our results, we suggest that baicalin could be a potential agent of treatment of acute lung injury. 


\section{Acknowledgement}

We thank Prof. Wang, Dao-Xin (Second Affiliated of Chongqing Medical University, China) for invaluable advice and discussions. This study was supported by grants from Medical Science Foundation of Chongqing Health and Family Planning Commission (Grant No. 2015ZBXM042).

\section{Footnotes}

Conflict of interest: none declared 


\section{References}

Baines, D.L., Albert, A.P., Hazell, M.J., Gambling, L., Woollhead, A.M., and Dockrell, M.E. 2010. Lipopolysaccharide modifies amiloride-sensitive $\mathrm{Na}+$ transport processes across human airway cells: role of mitogen-activated protein kinases ERK 1/2 and 5. Pflugers. Arch. 459(3): 451-463.

Benos, D.J., Saccomani, G., and Sariban-Sohraby, S. 1987. The epithelial sodium channel. Subunit number and location of the amiloride binding site. J. Biol. Chem. 262(22): 10613-10618.

Canessa, C.M., Horisberger, J.D., and Rossier, B.C. 1993. Epithelial sodium channel related to proteins involved in neurodegeneration. Nature, 361(6411): 467-470.

Canessa, C.M., Schild, L., Buell, G., Thorens, B., Gautschi, I., Horisberger, J.D. et al. 1994.

Amiloride-sensitive epithelial $\mathrm{Na}+$ channel is made of three homologous subunits. Nature, 367

(6462): 463-467.

Cheng, K.T., Hou, W.C., Huang, Y.C. and Wang, L.F. 2003. Baicalin induces differential expression of cytochrome C oxidase in human lung H441 cell. J. Agric. Food Chem. 51(25):

7276-7279.

Dagenais, A., Denis ,C., Vives, M.F., Girouard, S., Massé, C., Nguyen, T., et al. 2001. Modulation of alpha-ENaC and alpha1-Na+-K+-ATPase by cAMP and dexamethasone in alveolar epithelial cells. Am. J. Physiol. Lung Cell Mol. Physiol. 281(1): 217-230.

Dagenais, A., Gosselin, D., Guilbault, C., Radzioch, D., and Berthiaume, Y. 2005. Modulation of epithelial sodium channel $(\mathrm{ENaC})$ expression in mouse lung infected with Pseudomonas aeruginosa. Respir. Res. 6: 2-10.

Ding, X.M., Pan, L., Wang, Y., and Xu, Q.Z. 2016. Baicalin exerts protective effects against lipopolysaccharide-induced acute lung injury by regulating the crosstalk between the CX3CL1-CX3CR1 axis and NF-кB pathway in CX3CL1-knockout mice. Int. J. Mol. Med. 37(3): 703-715.

Dodrill, M.W., and Fedan, J.S. 2010. Lipopolysaccharide hyperpolarizes guinea pig airway epithelium by increasing the activities of the epithelial $\mathrm{Na}(+)$ channel and the $\mathrm{Na}(+)-\mathrm{K}(+)$ pump. Am. J. Physiol. Lung Cell Mol. Physiol. 299(4): 550-558.

Hassoun, P.M., Yu, F.S., Cote, C.G., Zulueta, J.J., Sawhney, R., Skinner, K.A., et al. 1998. Lanzillo JJ. Upregulation of xanthine oxidase by lipopolysaccharide, interleukin-1, and hypoxia. Role in acute lung injury. Am. J. Respir. Crit. Care Med. 158(1): 299-305.

Hummler, E., Barker, P., Gatzy, J., Beermann, F., Verdumo, C., Schmidt, A., et al. 1996. Early death due to defective neonatal lung liquid clearance in alpha-ENaC-deficient mice. Nat. Genet. 12(3): 325-328.

Johnson, M.D., Bao, H.F., Helms, M.N., Chen, X.J., Tigue, Z., Jain, L., et al. 2006. Functional ion channels in pulmonary alveolar type I cells support a role for type I cells in lung ion transport. Proc. Natl. Acad. Sci. U.S.A. 103(13): 4964-4969.

Lee, W., Ku, S.K., and Bae, J.S. 2015. Antiplatelet, anticoagulant, and profibrinolytic activities of baicalin. Arch. Pharm. Res. 38(5): 893-903.

Li, M.H., Huang, K.L., Wu, S.Y., Chen, C.W., Yan, H.C., Hsu, K., et al. 2009. Baicalin attenuates air embolism-induced acute lung injury in rat isolated lungs. Br. J. Pharmacol. 157(2): 244-251.

Lin, Y.L., Dai, Z.K., Lin, R.J., Chu, K.S., Chen, I.J., Wu, J.R., et al. 2010. Baicalin, a flavonoid 
from Scutellaria baicalensis Georgi, activates large-conductance $\mathrm{Ca} 2+$-activated $\mathrm{K}+$ channels via cyclic nucleotide-dependent protein kinases in mesenteric artery. Phytomedicine, 17(10): 760-770. Lingueglia, E., Voilley, N., Lazdunski, M., and Barbry, P. 1996. Molecular biology of the amiloride- sensitive epithelial Na+ channel. Exp. Physiol. 81(3): 483-492.

Liu, I.X., Durham, D.G., and Richards, R.M. 2000. Baicalin synergy with beta-lactam antibiotics against methicillin-resistant Staphylococcus aureus and other beta-lactam-resistant strains of S. aureus. J. Pharm. Pharmacol. 52(3): 361-366.

Liu, L.L., Gong, L.K., Wang, H., Xiao, Y., Wu, X.F., Zhang, Y.H., et al. 2008. Baicalin inhibits macrophage activation by lipopolysaccharide and protects mice from endotoxin shock. Biochem. Pharmacol. 75(4): 914-922.

Lucas, R., Verin, A.D., Black, S.M., and Catravas, J.D. 2009. Regulators of endothelial and epithelial barrier integrity and function in acute lung injury. Biochem. Pharmacol. 77 (12): 1763-1772.

Minakata, Y., Suzuki, S., Grygorczyk, C., Dagenais, A., and Berthiaume, Y. 1998. Impact of beta-adrenergic agonist on $\mathrm{Na}+$ channel and $\mathrm{Na}+-\mathrm{K}+$-ATPase expression in alveolar type II cells. Am. J. Physiol. 275(2): 414-422.

Morris, R.G. and Schafer, J.A. 2002. cAMP increases density of ENaC subunits in the apical membrane of MDCK cells in direct proportion to amiloride-sensitive $\mathrm{Na}(+)$ transport. J. Gen. Physiol. 120(1): 71-85.

Mustafa, S.B., Castro, R., Falck, A.J., Petershack, J.A., Henson, B.M., Mendoza, Y.M., et al. 2008. Protein kinase $\mathrm{A}$ and mitogen-activated protein kinase pathways mediate cAMP induction of alpha-epithelial Na+ channels (alpha-ENaC). J. Cell Physiol. 215(1): 101-110.

Otulakowski, G., Rafii, B., Bremner, H.R., and O'Brodovich, H. 1999. Structure and hormone responsiveness of the gene encoding the alpha-subunit of the rat amiloride-sensitive epithelial sodium channel. Am. J. Respir. Cell Mol. Biol. 20(5): 1028-1040.

Phua, J., Badia, J.R., Adhikari, N.K., Friedrich, J.O., Fowler, R.A., Singh, J.M., et al. 2009. Has mortality from acute respiratory distress syndrome decreased over time? A systematic review. Am. J. Respir. Crit. Care Med. 179(3): 220-227.

Planès, C., and Caughey, G.H. 2007. Regulation of the epithelial Na+ channel by peptidases. Curr. Top. Dev. Biol. 78: 23-46.

Richards, J.S. 2001. New signaling pathways for hormones and cyclic adenosine 3',5'-monophosphate action in endocrine cells. Mol. Endocrinol. 15(2): 209-218.

Sakuma, T., Zhao, Y., Sugita, M., Sagawa, M., Toga, H., Ishibashi, T., et al. 2004. Malnutrition impairs alveolar fluid clearance in rat lungs. Am. J. Physiol. Lung Cell Mol. Physiol. 286(6): 1268-1274.

Sato, K., Suga, M., Akaike, T., Fujii, S., Muranaka, H., Doi, T., et al. 1998. Therapeutic effect of erythromycin on influenza virus-induced lung injury in mice. Am. J. Respir. Crit. Care Med. 157(3): 853-857.

Seybold, J., Newton, R., Wright, L., Finney, P.A., Suttorp, N., Barnes, P.J., et al. 1998. Induction of phosphodiesterases 3B, 4A4, 4D1, 4D2, and 4D3 in Jurkat T-cells and human peripheral blood T-lymphocytes by 8-bromo-cAMP and Gs-coupled receptor agonists. Potential role in B2-adrenoreceptor desensitization. J. Biol. Chem. 273: 20575-20588.

Sheng, S.J., Nie, Y.C., Lin, F., Li, P.B., Liu, M.H., Xie, C.S., et al. 2014. Biphasic modulation of $\alpha$-ENaC expression by lipopolysaccharide in vitro and in vivo. Mol. Med. Rep. 10(2): 773-777. 
Snyder, P.M. 2000. Liddle's syndrome mutations disrupt cAMP-mediated translocation of the epithelial $\mathrm{Na}(+)$ channel to the cell surface. J. Clin. Invest. 105(1): 45-53.

Tang, B.M., Craig, J.C., Eslick, G.D., Seppelt, I., and McLean, A.S. 2009. Use of corticosteroids in acute lung injury and acute respiratory distress syndrome: a systematic review and meta-analysis. Crit. Care Med. 37(5): 1594-1603.

Thomas, C.P., Campbell, J.R., Wright, P.J., and Husted, R.F. 2004. cAMP-stimulated Na+ transport in H441 distal lung epithelial cells: role of PKA, phosphatidylinositol 3-kinase, and sgk1. Am. J. Physiol. Lung Cell Mol. Physiol. 287(4): 843-851.

Vivek, B., and Kenneth, R.H. 2008. Mechanisms of ENaC regulation and clinical implications. J. Am. Soc. Nephrol. 19: 1845-1854.

Vossler, M.R., Yao, H., York, R.D., Pan, M.G., Rim, C.S., and Stork, P.J. 1997. cAMP activates MAP kinase and Elk-1 through a B-Raf- and Rap1-dependent pathway. Cell, 89(1): 73-82.

Wang, H., and Liu, D. 2014. Baicalin inhibits high-mobility group box 1 release and improves survival in experimental sepsis. Shock, 41(4):324-330.

Ware, L.B., and Matthay, M.A. 2001. Alveolar fluid clearance is impaired in the majority of patients with acute lung injury and the acute respiratory distress syndrome. Am. J. Respir. Crit. Care Med. 163(6): 1376-1383.

Zhang, X.P., Zhang, L., Yang, P., Zhang, R.P., and Cheng, Q.H. 2008. Protective effects of baicalin and octreotide on multiple organ injury in severe acute pancreatitis. Dig. Dis. Sci. 53(2): 581-591. 
Figure legends

Figure 1 Effects of baicalin on rat lung water content.

SD rats received several doses of Baicalin $(10,50$ and $100 \mathrm{mg} / \mathrm{kg}$ ) for 6 hours ( $n=10$ per group). Then lungs were isolated and dried at $95^{\circ} \mathrm{C}$ for 48 hours. Lung water content was estimated by calculating the ratio of the wet lung weight to the dry lung weight per gram (W/D ratio) of body weight. Mean values \pm sem. $\triangle p<0.01$ vs control. $* p<0.05$ vs LPS. $\# p<0.01$ vs LPS.

Figure 2 Effects of baicalin on cyclic adenosine monophosphate (cAMP) concentration in lung.

Rats were given LPS or baicalin $(10,50$ and $100 \mathrm{mg} / \mathrm{kg})$ for 6 hours, and cAMP in lung was determined by RIA ( $\mathrm{n}=30$ per group). Mean values \pm sem. $\triangle p<0.01$ vs control. $* p<0.05$ vs LPS.

Figure 3 Effects of bacalin on rat alveolar fluid clearance (AFC).

SD rats received increasing doses of baicalin $(10,50$ and $100 \mathrm{mg} / \mathrm{kg}$ ) before administration of LPS ( $n=10$ per group). Then lungs were isolated, and instilled with evans-blue labeled albumin (4 $\mathrm{mL} / \mathrm{kg}$ ). AFC was measured 1 hour after ventilation. Mean values \pm sem. $\triangle p<0.01$ vs LPS. * $p<0.05$ vs Baicalin $10 \mathrm{mg} / \mathrm{kg}$. \# $p<0.05$ vs Baicalin $50 \mathrm{mg} / \mathrm{kg}$.

Figure 4 Effects of amiloride, LPS and baicalin on rat alveolar fluid clearance (AFC). SD rats received LPS or Baicalin $(100 \mathrm{mg} / \mathrm{kg})$ for 6 hours. Then AFC was measured 1 hour after fluid instillation $(4 \mathrm{~mL} / \mathrm{kg})$. Amiloride $(100 \mu \mathrm{M})$ was added to the instillate as indicated $(n=10$ per group). Mean values \pm sem. $\triangle p<0.01$ vs control. $* p<0.05$ vs LPS. $\triangle p<0.05$ vs amiloride. $\# p<0.01$ vs LPS+Baicalin.

Figure 5 Histological examination of lung.

Rats were given saline or LPS for 6 hours. Baicalin $(100 \mathrm{mg} / \mathrm{kg})$ was injected intraperitoneally 30 min before administration of LPS. Shown are representative lung specimens obtained from the control (A), LPS (B) and baicalin pre-treatment (C) groups. All photographs are at $\times 100$ magnification. Interstitial edema and inflammatory cell infiltration were seen in LPS group, but reduced in baicalin pre-treatment groups.

Figure 6 Changes in $\alpha$-ENaC protein expression in lung tissue specimens.

Rats were given saline or LPS for 6 hours. Baicalin $(100 \mathrm{mg} / \mathrm{kg})$ was injected intraperitoneally 30 min before administration of LPS. Immunohistochemical analysis was used to detect $\alpha$-ENaC in lung sections. Representative specimens from the control (A), LPS (B) and LPS + Baicalin (C) groups are presented. The number of positive cells was counted in randomly 5 high-power fields of each section and averaged. All photographs are at $\times 400$ magnification. The number of cells expressing $\alpha$-ENaC decreased after LPS administration, but increased in the baicalin pre-treatment group.

Figure 7 Relative expression of $\alpha$-epithelial sodium channel ( $\alpha$-ENaC) induced by baicalin, 
SQ22536 or KT5720 in vitro.

ATII cell was cultured in high DMEM (Hyclone) with 10\% FBS (GIBCO), 1\% L-glutamine, and a $1 \%$ solution of penicillin and streptomycin or the same medium supplemented with baicalin (30uM), SQ22536 (10uM) or KT5720 (0.3uM) $15 \mathrm{~min}$ before LPS $(1 \mu \mathrm{g} / \mathrm{ml})$ incubation. The protein expression of $\alpha$-ENaC was determined by Western blot (left panel). Data was shown in right panel ( $n=5$ per group). $\triangle p<0.01$ vs control. $* p<0.05$ vs LPS. $\triangle p<0.05$ vs LPS. \# $p<0.05$ vs LPS+Baicalin. 


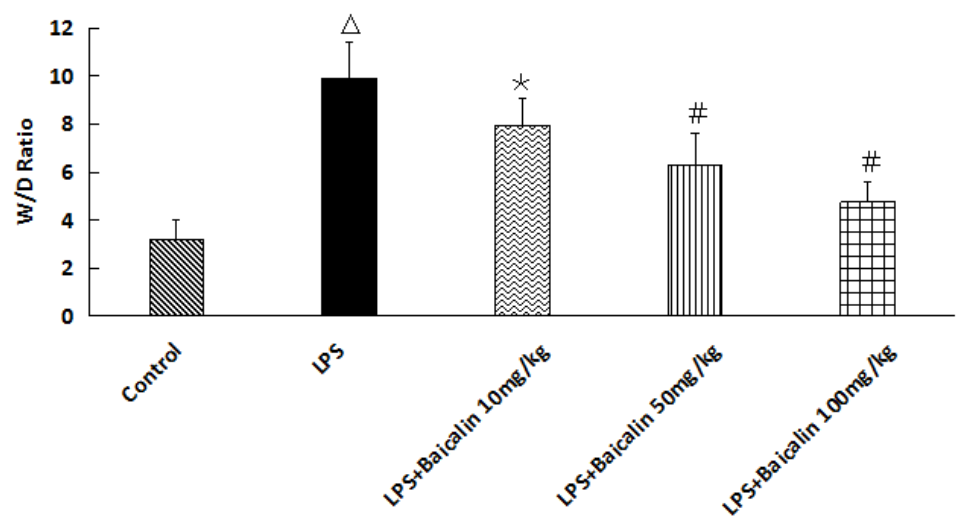

Figure 1 


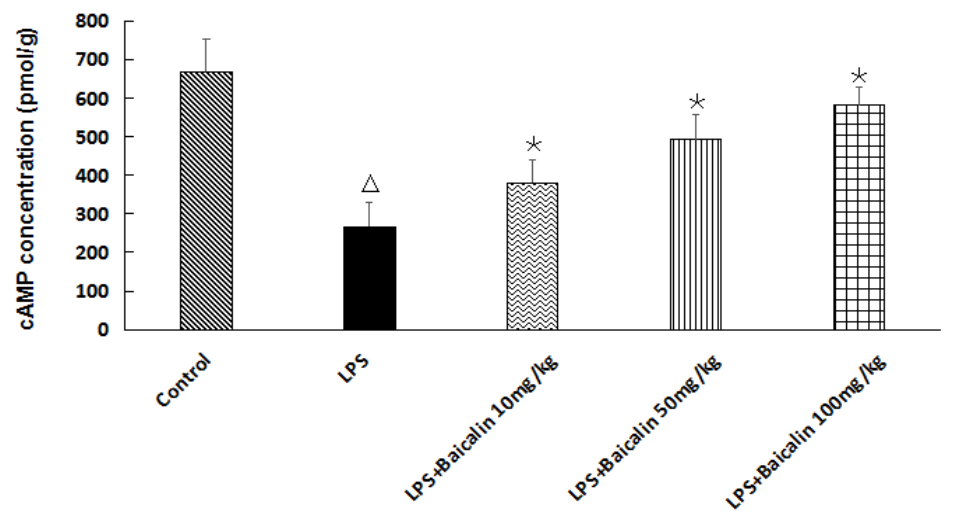

Figure 2 


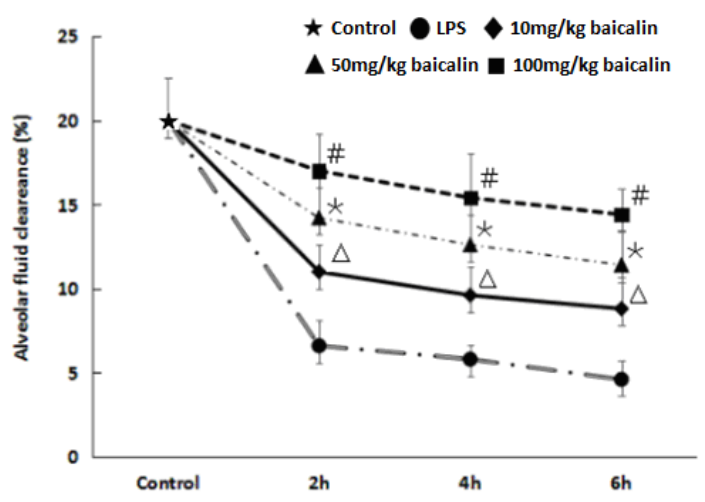

Figure 3 


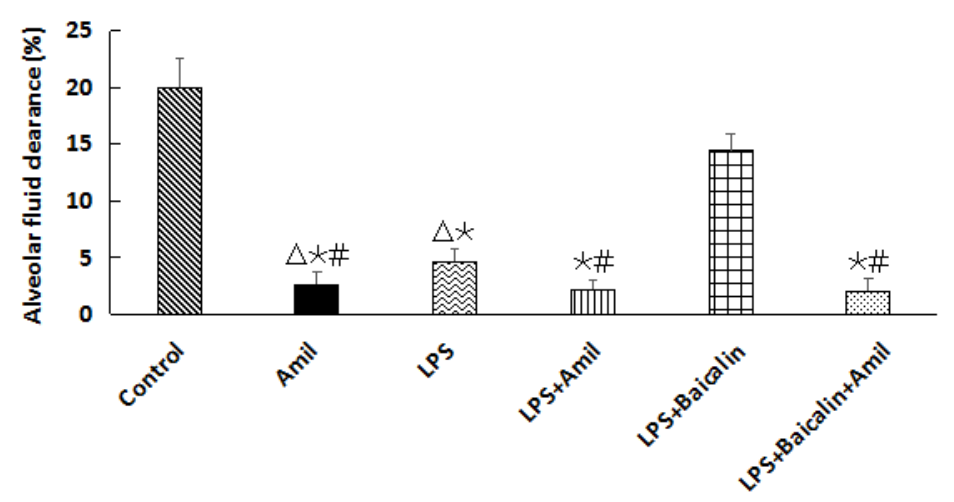

Figure 4 
A

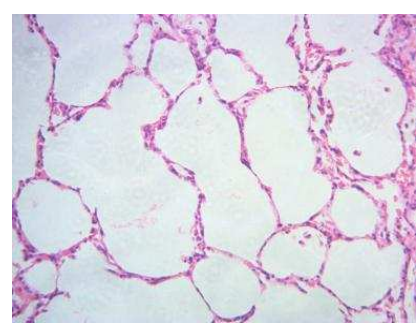

B

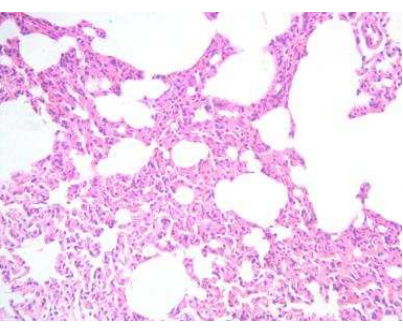

C

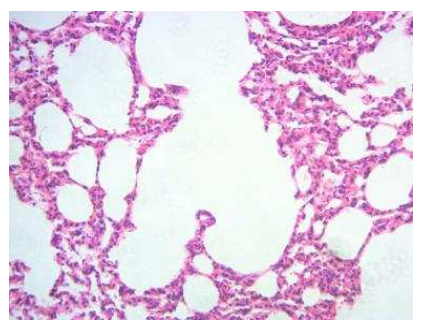

Figure 5 

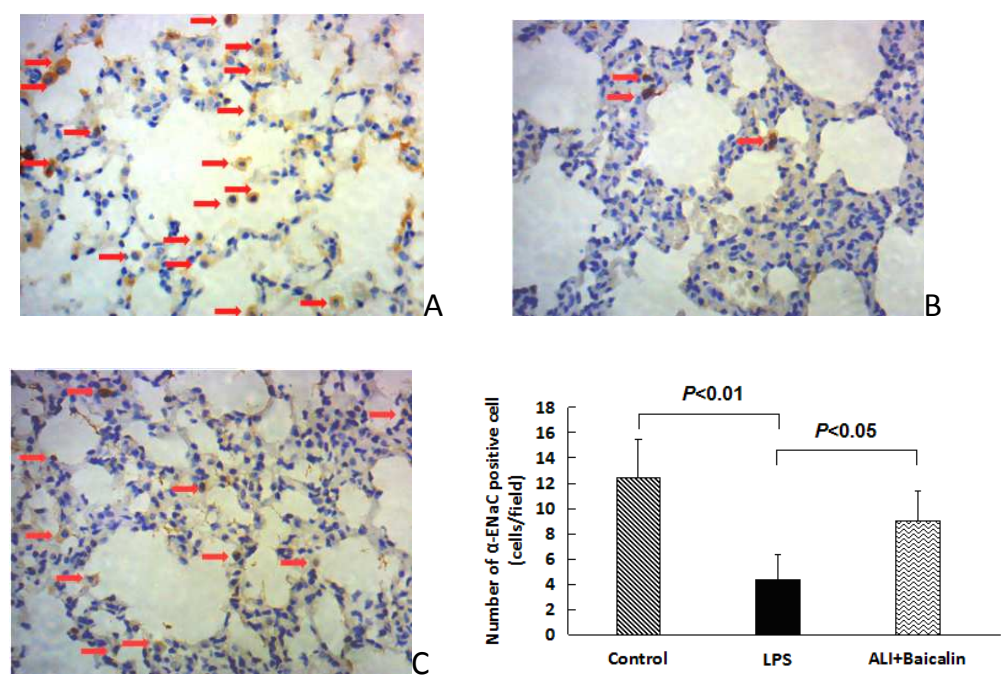

Figure 6 

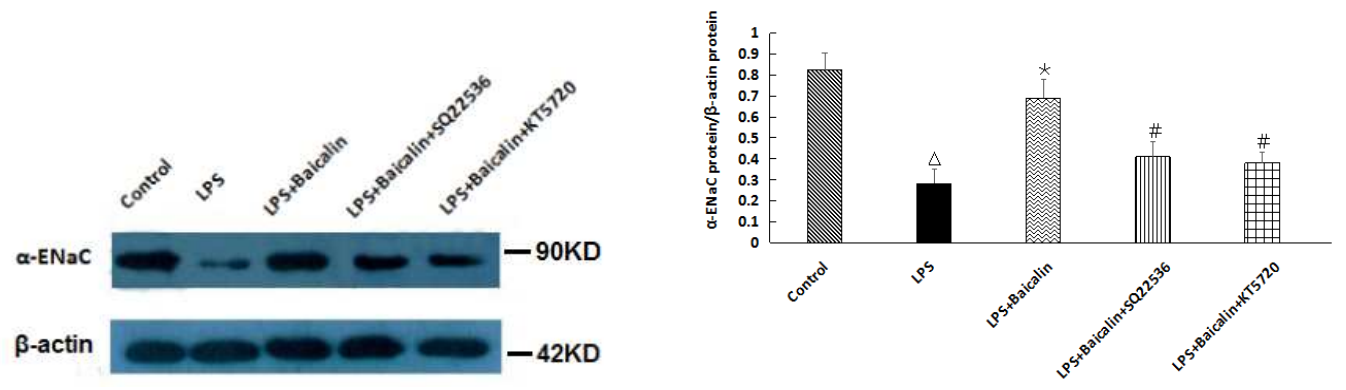

Figure 7

https://mc06.manuscriptcentral.com/cjpp-pubs 